\title{
Distribución del cobre en ecosistemas forestales de tipo mediterraneo
}

\author{
Copper distribution in Mediterranean forest ecosystems
}

\author{
LUISA DELGADO ${ }^{1} \&$ ITALO SEREY
}

Laboratorio de Modelación Ecológica, Departamento de Ciencias Ecológicas, Facultad de Ciencias, Universidad de Chile, Casilla 653, Santiago, Chile; 'e-mail: ldelgado@antar.uchile.cl

\begin{abstract}
RESUMEN
En este estudio hemos usado el método comparativo para analizar la distribución de la concentración total de cobre en los componentes de dos ecosistemas forestales, y el efecto del ingreso de cobre antropogénico. Los componentes ecosistémicos se definieron a partir de un modelo conceptual basado en la aproximación proceso-funcional. Los componentes bióticos correspondieron a las estructuras morfológicas de los árboles; en tanto que los abióticos a los distintos horizontes del suelo. Se escogieron dos áreas que difieren en cuanto a la ausencia (ecosistema de referencia) y presencia (ecosistema perturbado) de ingreso de cobre antropogénico generado por la Refinería de VentanasMaitencillo. Los resultados muestran que el cobre antropogénico es retenido en el material parcialmente descompuesto, y que los componentes bióticos del ecosistema perturbado presentan concentraciones de cobre por sobre los valores normales para ecosistemas forestales. Se discuten las variables controladoras del ciclo intrasistémico del cobre en ecosistemas forestales.
\end{abstract}

Palabras clave: aproximación proceso-funcional, estudio comparativo, cobre, ecosistemas forestales.

\begin{abstract}
We have analyzed the distribution of copper concentrations, and the effects of anthropogenic inputs, in the components of two forest ecosystems using a comparative approach. Ecosystem components were defined using a processfunctional conceptual model. Biotic components corresponded to the morphological structures of trees, the abiotic components to the soil horizons. We chose two areas that differed on the absence (reference ecosystem) and presence (perturbed ecosystem) of an anthopogenic copper source (the Ventanas-Maitencillo Refinery). Results show that the anthropogenic copper is mostly retained in the partially decomposed matter, and that the biotic components of the perturbed ecosystem show higher that normal copper concentrations. We discuss the controlling variables of the intrasystemic copper cycle in forest ecosystems.
\end{abstract}

Key words: process-functional approach, comparative study, copper, forest ecosystems.

\section{INTRODUCCIÓN}

La concentración y distribución de minerales en ecosistemas forestales están determinadas por tres fuentes: abiótica (geológica, atmosférica, hidrológica), biótica (mineralización de material orgánico), y antrópica (actividades industriales). Las fuentes antrópicas, debido especialmente a la contaminación por metales pesados, han recibido mucha atención (Zhou \& Wong 2001). Aún así, existe poca información sobre la concentración y la distribución de micronutrientes en los componentes de los ecosistemas forestales (Keller \& Védy 1994). Uno de estos micronutrientes, el cobre, ha sido particularmente poco estudiado pese a su frecuente uso industrial y a la necesidad de recuperar sitios altamente contaminados (Kramer et al. 2000).
Los estudios sobre el efecto de un exceso de cobre en ecosistemas forestales han sido realizados desde una perspectiva fisiológica (e.g., inhibición del crecimiento [Filani 1976] y reproducción [Cox 1988]) o de análisis de un solo componente, generalmente el suelo. En este último caso se incluye, perturbación de la descomposición biológica de la hojarasca y materia húmica del suelo (Løbersli \& Steinnes 1988) y la acumulación de cobre en la materia orgánica (Zhou \& Wong 2001). Una aproximación alternativa a las anteriores es analizar los efectos del cobre en ecosistemas forestales considerando a éstos como sistemas parcialmente autónomos con retroalimentación positiva (Ulanowicz 1987, 1991, Jørgensen \& Müller 2000). Desde esta perspectiva, la distribución de un elemento en un ecosistema dependerá de las interacciones entre sus compo- 
nentes. Lo anterior puede evaluarse, en una primera instancia, estudiando la distribución del elemento en los diversos componentes que forman el ecosistema bajo estudio. Pese a los problemas de contaminación derivados de emisiones atmosféricas, tales como lluvia ácida, depositación de cobre particulado así como de relaves industriales líquidos procedentes de las actividades mineras, los estudios ecosistémicos relativos al cobre en Chile son escasos.
Uno de los principales problemas en el uso de aproximaciones ecosistémicas reside en la variedad de conceptos y bases filosóficas asociadas (e.g., Tansley 1935, Likens 1992, Blew 1996, Marín 1996, Fauth 1997, Rowe 1997, Barkmann et al. 1998). Nosotros usamos en este estudio el concepto propuesto por Marín (1996), el cual está basado en la teoría general de sistemas. Vistos desde esta perspectiva, los ecosistemas son unidades conceptuales adimensionales que consisten

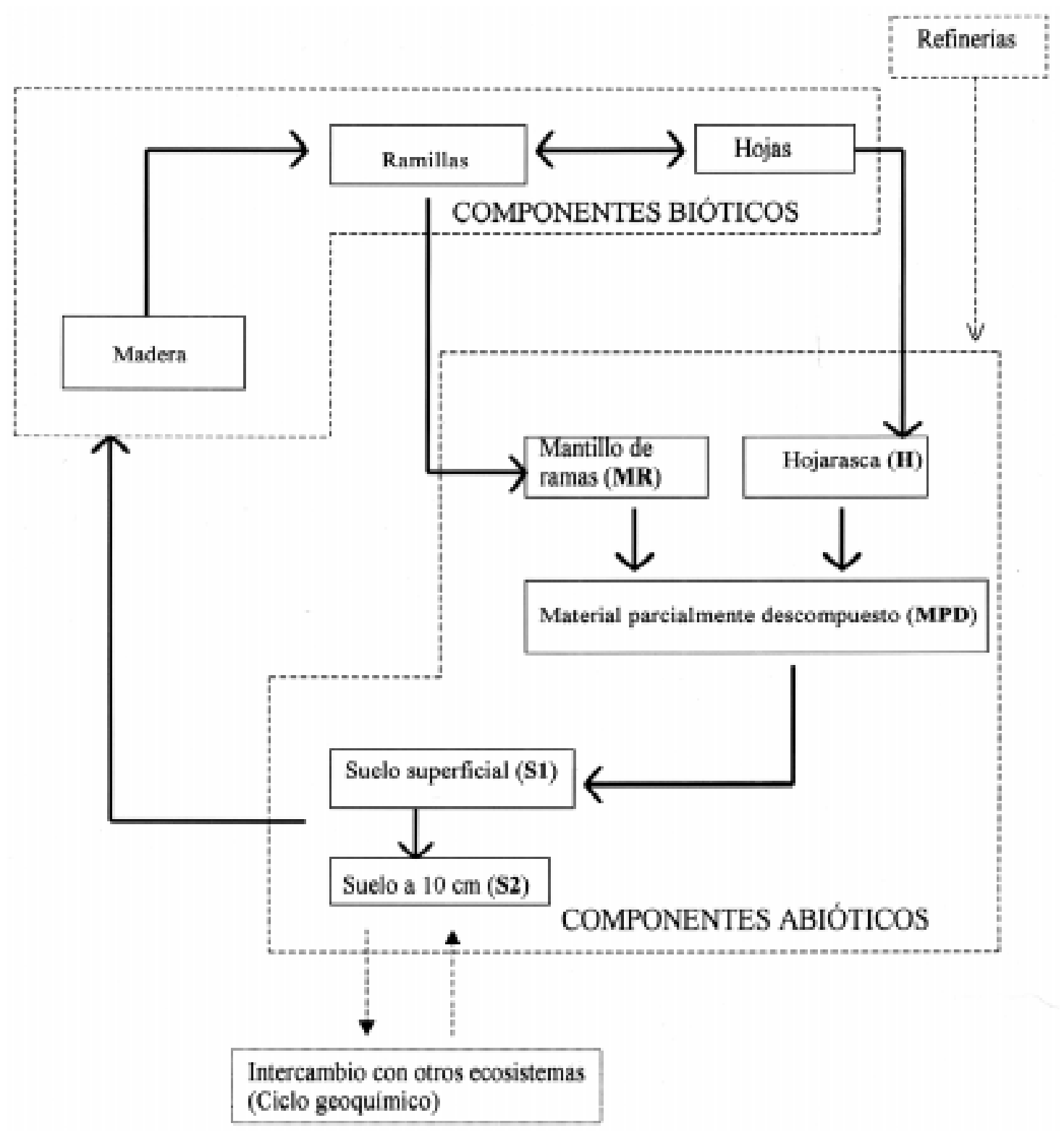

Fig. 1: Ciclo intrasistémico del cobre en un ecosistema forestal (modificado de Serey et al. 1997). Los componentes bióticos se diferencian por sus tasas de mortalidad y descomposición; los componentes abióticos se diferencian por su grado de descomposición y mineralización.

Intrasistemic cycle of copper in a forest ecosystem (modified from Serey et al. 1997). Biotic components are differentiated by their mortality and decomposition rates; abiotic components are differentiated by their degree of decomposition and mineralization. 
en un conjunto de componentes bióticos y abióticos interactivos, cuya delimitación depende del objetivo del estudio. Una vez que los objetivos y los límites han sido establecidos, el sistema así definido puede ser analizado como un sistema ecológico jerárquico y multiescalar (Jørgensen 1992).

Existen dos aproximaciones para definir los componentes de un ecosistema: (1) poblacionalcomunitaria, que considera los ecosistemas como conjuntos de especies y (2) proceso-funcional, que no restringe los componentes a especies biológicas, pero que requiere definirlos respecto de un proceso ecológico específico (O'Neill et al. 1986, Marín \& Olivares 1999). La definición de los componentes depende, además, si el estudio es intra o intersistémico (Duvigneaud \& DenaeyerDe Semet 1970, Waring \& Schlesinger 1985). En este estudio hemos definido los componentes del ciclo intrasistémico del cobre, en bosques mediterráneos de Chile Central, basados en la aproximación proceso-funcional. Para ello elaboramos un modelo conceptual de este ciclo, a partir del cual se definieron los componentes a analizar. El objetivo principal de este trabajo fue estudiar el contenido total de cobre en los componentes de un ecosistema forestal mediterráneo en Chile Central, definir su estructura y proponer mecanismos de transferencia y control.

\section{MATERIALES Y MÉTODOS}

\section{Selección de áreas de estudio}

En este trabajo se utilizó el método comparativo (Mooney et al. 1991), seleccionando para ello dos áreas geográficas diferenciadas sobre la base de la presencia (ecosistema perturbado) y ausencia (ecosistema de referencia) de enriquecimiento antropogénico de cobre.

El ecosistema perturbado se ubica en la zona de influencia de la refinería de cobre en Ventanas $\left(31^{\circ} 43^{\prime} \mathrm{S}, 71^{\circ} 22^{\prime} \mathrm{O}\right)$. Esta área se encuentra afectada por deposición atmosférica de partículas provenientes de la chimenea de esta refinería por más de treinta años. El ecosistema de referencia se seleccionó luego de muestreos preliminares en los que se determinó la concentración de cobre en hojas en distintas localidades. La selección de este componente estuvo basada en nuestro modelo conceptual del ciclo intrasistémico del cobre (Fig. 1). Esto es, el cobre antropogénico debe haber circulado al menos una vez a través del ecosistema para alcanzar esta estructura. El área seleccionada fue Quintay $\left(33^{\circ} 12^{\prime} \mathrm{S}, 71^{\circ} 30^{\prime} \mathrm{O}\right)$,
$60 \mathrm{~km}$ al sur de la refinería de Ventanas. Esta no recibe aportes atmosféricos de cobre debido a que la dirección del viento dominante en la zona es NE debido a la influencia del anticiclón del Pacífico Sur Oriental. Las áreas seleccionadas son similares en cuanto a su composición florística, origen del suelo y clima (Donoso 1993). El bosque es de tipo esclerófilo, con una vegetación fragmentada y dominada por árboles de Cryptocarya alba (Lauraceae), que constituye más del $80 \%$ de la cobertura total del ecosistema forestal.

\section{Ciclo intrasistémico del cobre}

La Fig. 1 muestra nuestro modelo conceptual de las transferencias intrasistémicas del cobre en bosques mediterráneos. El modelo representa el intercambio de cobre entre el suelo (componentes abióticos) y la vegetación (componentes bióticos). El cobre antropogénico ingresa al ecosistema (Refinerías en Fig. 1) a través de los componentes abióticos en los cuales, por medio del proceso de mineralización, se hace disponible a los demás componentes. Los componentes bióticos corresponden a estructuras morfológicas de los árboles, diferenciadas sobre la base de sus tasas de mortalidad y descomposición (Harper 1977, Hallé et al. 1978), las cuales están directamente relacionadas a sus características morfofisiológicas y composición química (Pérez 1994). Estas últimas características, a su vez, influyen en la tasa de descomposición de la hojarasca y el aporte de nutrientes a la solución del suelo. En este modelo conceptual no hemos incluido las raíces por razones metodológicas, ya que solo deberían considerarse las más finas, que son difíciles de separar del suelo.

Los componentes abióticos, hojarasca, mantillo de ramas, material parcialmente descompuesto y suelo mineral (superficial y $10 \mathrm{~cm}$ ), se diferenciaron sobre la base de su grado de descomposición y mineralización (Zunino \& Martin 1977, Waring \& Schlesinger 1985). Estos constituyen los componentes más activos fisicoquímicamente, y en ellos están centrados los procesos que proveen el cobre para la vegetación.

Muestreo y determinación del cobre en los componentes ecosistémicos

La unidades de muestreo en este estudio la constituyeron los ecosistemas de Ventanas y Quintay. En cada ecosistema se muestrearon, una sola vez y en la misma oportunidad, siete sitios (pseudo- 
réplicas), en los cuales se colectaron muestras de todos los componentes (Fig. 1). Este número de sitios se determinó calculando el número mínimo de réplicas (Sokal \& Rohlf 1981) usando la información disponible sobre la variabilidad en los contenidos de cobre en otros ecosistemas forestales mediterráneos (Serey et al. 1997). Todas las muestras se colectaron durante la primavera de 1995. Se recolectaron $50 \mathrm{~g}$ de cada componente; en la recolección se utilizaron guantes desechables estériles (Vinyl Exam Gloves) con objeto de no contaminar las muestras. Los componentes vivos fueron lavados con agua destilada-desmineralizada para eliminar partículas adheridas a la superficie. Todas las muestras fueron secadas por 48 horas, homogeneizadas y tamizadas en tamices Soiltest de $2 \mu \mathrm{m}$. La digestión de las muestras, tres réplicas analíticas de cada componente, se realizó usando una mezcla nítrico-sulfúrico $(3: 1)$ a $350^{\circ} \mathrm{C}$, previo secado a peso constante $( \pm 0,01$ g). Este procedimiento destruye los enlaces entre el cobre y sus ligandos permitiendo estimar la concentración total de cobre en la muestra como $\mathrm{Cu}^{+2}$. La lectura se realizó por el método de espectrofotometría de absorción atómica (Besier et al. 1994), usando un espectrofotómetro PerkinElmer modelo 360, el cual fue calibrado con estándares de cobre conocido (High Purity Standars, Laboratorios Merck).

\section{Enriquecimiento y coeficientes de trasferencia del cobre}

Para calcular el enriquecimiento antropogénico de cobre en el ecosistema perturbado se determinó un coeficiente promedio de enriquecimiento, $\overline{\mathrm{K}}_{\mathrm{i}}$, por medio de la siguiente fórmula:

$$
\overline{\mathrm{K}}_{\mathrm{i}}=\frac{\overline{\mathrm{Cu}}_{\mathrm{c}, \mathrm{i}}}{\overline{\mathrm{Cu}}_{\mathrm{p}, \mathrm{i}}}
$$

Donde, $\overline{\mathrm{Cu}}_{\mathrm{c}, \mathrm{i}}$ es la concentración promedio de cobre en el componente i, en el ecosistema perturbado y $\overline{\mathrm{Cu}}_{\mathrm{p}, \mathrm{i}}$ es la concentración promedio en el ecosistema de referencia. Los valores $\overline{\mathrm{K}}_{\mathrm{i}}$ se calcularon comparando los valores de todas las muestras entre ambos ecosistemas. La significacia de las diferencias entre los valores $\overline{\mathrm{K}}_{\mathrm{i}}$ se determinó por medio de un análisis de varianza paramétrico de una vía.

Como una forma de estimar las diferencias relativas en biodisponibilidad de cobre se determinó el coeficiente de transferencia CT (Alloway \& Ayres 1993), que se define como la concentración de algún metal en la parte aérea de la planta relativa a la concentración en el suelo. Con el propósito de comparar los valores obtenidos con aquellos disponibles en la literatura (Alloway \& Ayres 1993), se determinó el promedio de la concentración de cobre en los componentes bióticos aéreos (hojas y ramillas) relativa al promedio de la concentración de cobre en los componentes del suelo (S1 y S2). Así también para obtener una estimación de la transferencia del cobre entre el MPD y el suelo superficial (S1), se calculó la relación porcentual entre la concentración de cobre de ambos componentes (McBride 1994).

\section{RESULTADOS}

Distribución del cobre en los componentes de los ecosistemas

La concentración de cobre total para el ecosistema de referencia y el perturbado se presentan en la Tabla 1. Las mayores concentraciones, en ambos ecosistemas, se encontraron en los componentes abióticos. El menor valor promedio (2,6 ppm) se encontró en la madera del ecosistema de referencia, en tanto que el mayor valor (1.273,9 ppm) se encontró en el material parcialmente descompuesto, MPD, del ecosistema perturbado.

El cuociente entre la concentración de cobre de los componentes bióticos y abióticos, considerados de manera agregada (Fig. 1), fue levemente menor en el ecosistema perturbado ([ $\mathrm{Cu}$ biótico] / $[\mathrm{Cu}$ abiótico $]=0,08)$ que en el ecosistema de referencia ( $[\mathrm{Cu}$ biótico $] /[\mathrm{Cu}$ abiótico $]=0,13$ ). Sin embargo, este resultado está influenciado por la concentración de cobre en el material parcialmente descompuesto. Al considerar solo el suelo mineral como la fuente de cobre para los componentes bióticos, la relación en el ecosistema perturbado ([Cu biótico] / [Cu suelo mineral $]=0,27$ ) fue significativamente mayor a aquella del ecosistema de referencia ([Cu biótico] / [ $\mathrm{Cu}$ suelo mineral] $=0,12)$. El pH del suelo difirió significativamente $(\mathrm{P}=0,01)$ entre ambos ecosistemas, con un valor promedio de 6,6 en el ecosistema de referencia y de 5,8 en el ecosistema perturbado.

Las diferencias entre ambos ecosistemas, respecto de la concentración total de cobre y de su distribución entre los distintos componentes, se analizaron por medio de un ANDEVA de dos vías (Tabla 2). La prueba de homogeneidad de varianza (prueba de Bartlett, Sokal \& Rohlf 1982) mostró que existía heterogeneidad en las mismas, por lo que se usó la transformación logaritmo natural para estabilizarlas. El resultado mostró que hay diferencias significativas $(\mathrm{P}<0,001)$ entre los 


\section{TABLA 1}

Concentración de cobre (ppm) en los componentes del ecosistema de referencia y perturbado. Los valores corresponden al promedio y desviación estándar (DE), por componente, de los sietes sitios (pseudoréplicas) en cada ecosistema. Los componentes abióticos se identifican por su abreviación (ver Fig. 1)

Copper concentration ( $\mathrm{ppm}$ ) in the components of the reference and perturbed ecosystem. Values, per component, correspond to the average and standard deviation (DE) of the seven samples (pseudoreplicates) in each ecosystem. Abiotic components are identified by their abbreviation (see Fig. 1)

\begin{tabular}{lcccc}
\hline Componente & \multicolumn{2}{c}{ Ecosistema de referencia } & \multicolumn{2}{c}{ Ecosistema perturbado } \\
& Promedio & DE & Promedio & DE \\
\hline Madera & 2,62 & 0,89 & 8,20 & 3,45 \\
Ramillas & 10,61 & 2,97 & 74,06 & 21,64 \\
Hojas & 3,67 & 0,96 & 26,75 & 10,05 \\
H & 12,53 & 6,88 & 230,124 & 77,67 \\
MR & 29,82 & 191,35 & 408,00 & 98,71 \\
MPD & 86,14 & 14,73 & 1273,88 & 268,62 \\
S1 & 51,78 & 22,29 & 196,33 & 102,90 \\
S2 & 44,35 & 22,14 & 69,95 & 24,97 \\
\hline
\end{tabular}

ecosistemas, entre los componentes así como en la distribución del cobre en los componentes (interacción). El análisis de estas diferencias se realizó por medio del cálculo de coeficientes de enriquecimiento.

\section{Enriquecimiento y coeficientes de trasferencia del cobre}

El enriquecimiento en cobre en el ecosistema perturbado, estimado a través de los $\overline{\mathrm{K}}_{\mathrm{i}}$, fue altamente heterogéneo entre componentes. Los valores más elevados, esto es donde el enriquecimiento es mayor, correspondieron a la hojarasca y al mantillo de ramas, donde el $\overline{\mathrm{K}}_{\mathrm{i}}$ es cercano a 20 (Tabla 3). Es especialmente destacable que los componentes del suelo presentaron una disminución monotónica del enriquecimiento con la profundidad, con valores máximos cerca de superficie (hojarasca y mantillo de ramas) hasta valores mínimos $\left(\overline{\mathrm{K}}_{\mathrm{i}}=2,2\right)$ en los componentes más profundos (Fig. 2). Los componentes bióticos presentaron valores de enriquecimientos intermedios. El menor enriquecimiento correspondió a la madera $\left(\overline{\mathrm{K}}_{\mathrm{i}}=3,5\right)$, que presentó un valor cercano a los componentes abióticos S1 y S2. Las hojas y ramillas mostraron valores similares entre sí $\left(\overline{\mathrm{K}}_{\mathrm{i}}=7,5\right)$ y superiores a los del suelo mineral (Tabla 3).

TABLA 2

Análisis de varianza de dos vías para las concentraciones de cobre en los ecosistemas de referencia y perturbado

Two way analysis of variance of the copper concentration in the reference and perturbed ecosystem

\begin{tabular}{lccccc}
\hline Fuente de variación & $\begin{array}{c}\text { Suma de } \\
\text { cuadrados }\end{array}$ & $\begin{array}{c}\text { Grados de } \\
\text { libertad }\end{array}$ & $\begin{array}{c}\text { Cuadrado } \\
\text { medio }\end{array}$ & Valor de F & Valor de P \\
\hline Ecosistema & 97,23 & 1 & 97,23 & 449,26 & $<0,001$ \\
Componente & 174,98 & 7 & 24,99 & 115,49 & $<0,001$ \\
Ecosistema x componente & 18,58 & 7 & 2,65 & 12,27 & $<0,001$ \\
Error & 20,12 & 93 & 0,22 & & \\
\hline
\end{tabular}


TABLA 3

Promedio y desviación estándar (DE) de los coeficientes de enriquecimiento, $\mathrm{K}_{\mathrm{i}}$, para los componentes del ecosistema perturbado, respecto del ecosistema de referencia

Average and standard deviation (DE), of the enrichment coefficients, Ki, of the components of the perturbed respect to the reference ecosystem

\begin{tabular}{lcccccccc}
\hline & & \multicolumn{9}{c}{ Componente } \\
& Madera & Ramillas & Hojas & H & MR & MPD & S1 & S2 \\
\hline Promedio & 3,5 & 7,5 & 7,1 & 21,8 & 19,6 & 15,3 & 4,5 & 2,2 \\
DE & 2,2 & 3,0 & 3,0 & 10,2 & 12,0 & 4,4 & 3,1 & 1,6 \\
\hline
\end{tabular}

El análisis de la transferencia de cobre entre componentes, CT, mostró un valor promedio de $0,13(\mathrm{DE}=0,05)$ para el ecosistema de referencia. El valor para el ecosistema perturbado $(\mathrm{CT}=$ 0,$35 ; \mathrm{DE}=0,23$ ) fue significativamente superior $(\mathrm{P}<0,05)$ al del ecosistema de referencia y al rango normal para este metal. Por otra parte, los coeficientes de transferencia calculados separa- damente para los componentes bióticos mostraron que las ramillas presentan los mayores valores en ambos ecosistemas ( $\mathrm{CT}=0,22$ Quintay; $\mathrm{CT}$ $=0,56$ Ventanas $)$. El resultado de la transferencia del cobre entre el MPD y el suelo superficial (S1), muestra que esta sería de un $60 \%$ para el ecosistema de referencia y de solo un $16 \%$ para el ecosistema perturbado (Fig.2).

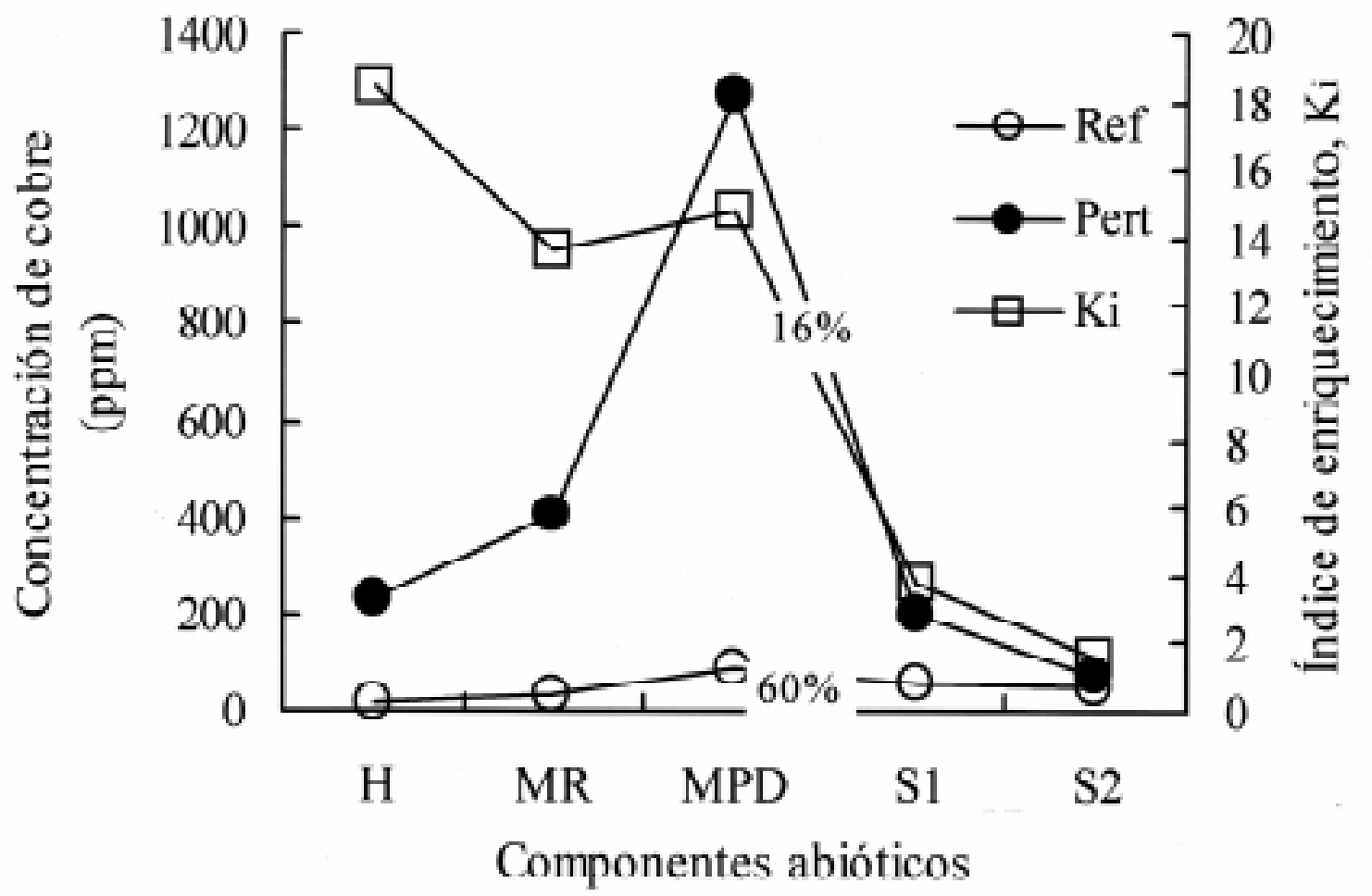

Fig. 2: Distribución de la concentración de cobre en los componentes abióticos del ciclo intrasistémico. Las abreviaciones para cada componente se encuentran en la Fig. 1. Las concentraciones del ecosistema de referencia (Ref) y del ecosistema perturbado (Pert) fueron usadas para calcular el índice de enriquecimiento (Ki). El porcentaje arriba de cada curva corresponde al cálculo de la transferencia de cobre entre el MPD y el S1 suponiendo que la fuente de cobre para el suelo fuera exclusivamente el MPD.

Distribution of copper concentration in the abiotic components of the intrasistemic cycle. Concentrations of the reference ecosystem (Ref) and perturbed ecosystem (Pert) were used to calculate the enrichment index (Ki). The percentage on top of each curve corresponds to the calculation of copper transference between the MPD and S1, assuming the MPD is the only source of copper. 


\section{DISCUSIÓN}

Existe poca información respecto del ingreso, movilidad, y retención de los micronutrientes como el cobre en ecosistemas forestales (Keller \& Védy 1994). Nuestros resultados muestran que la distribución de este elemento, en los componentes del ciclo intrasistémico de bosques mediterráneos en Chile central, depende en gran medida del pH del suelo y de la presencia de material parcialmente descompuesto, MPD. Ambos ecosistemas difieren en el pH del suelo (menor en el ecosistema perturbado), y se encuentran a ambos lados del límite teórico $(\mathrm{pH}=6,5)$ que separa la condición de inmovilización (ecosistema prístino) de aquella de liberación (ecosistema perturbado). De hecho, los coeficientes de transferencia CT del ecosistema perturbado se encuentran por sobre los valores considerados como normal (CT $\cong 0,1$ ) para el cobre (González 1986). Kiikilä et al. (2001) muestran que un aumento en el pH de la materia orgánica aumenta la complejación del cobre. Sin embargo, la transferencia de cobre entre el MPD y el suelo mineral fue menor en el ecosistema perturbado aún en presencia de un menor pH. Tyler (1984), en un estudio realizado en las cercanías de una refinería, ha mostrado que un exceso de cobre altera los procesos de descomposición y mineralización. Desafortunadamente, el conocimiento de la dinámica de los metales pesados en la descomposición de la hojarasca es particularmente escaso (Laskowski et al. 1995). La gran diferencia encontrada en la concentración de cobre del MPD entre ambos ecosistemas sugiere que mientras otros elementos, tales como el carbono y oxígeno, son liberados durante la descomposición (Laskowski et al. 1995), el cobre quedaría unido a las moléculas de las estructuras que quedan en el suelo (e.g., ácidos húmicos y fúlvicos) debido a su alta capacidad para formar complejos orgánicos estables (Lobersli \& Steinnes 1988, Stevenson 1994, Zhou \& Wong 2001). Esto estaría produciendo un aumento en la concentración en la hojarasca, lo que unido a la deposición atmosférica procedente de la refinería, genera las altas concentraciones observadas. Vale decir, una gran proporción del cobre antropogénico (superior a un $80 \%$ ) queda atrapado en los componentes del mantillo, sin transferirse proporcionalmente hacia los componentes más profundos del suelo. Esta característica del ciclo intrasistémico del cobre generaría las diferencias observadas entre las concentraciones de cobre en los componentes bióticos y abióticos.

Sin embargo, la distribución del cobre en los componentes bióticos no es homogénea. Las altas concentraciones encontradas en ramillas y hojas de Cryptocaria alba, especialmente en el ecosistema perturbado, así como la dominancia de esta en la zona de estudio sugieren que esta especie debe poseer mecanismos de de-toxificación los cuales deberían estudiarse. Ginnocchio (1996) propone, a partir de un estudio experimental de germinación y crecimiento de plántulas, que el cobre sería translocado de manera muy restringida desde las raíces hacia las estructuras vegetales aéreas. Sin embargo, nuestros resultados sugieren que, al menos después de un deposición antropogénica de cobre por un período cercano a los 30 años, las estructuras aéreas presentan concentraciones de cobre por sobre lo normal lo que sugiere translocación hacia dichas estructuras y potencialmente retención.

La modelación de los ciclos biogeoquímicos se ha llevado a cabo bajo dos supuestos alternativos: (1) que los flujos entre compartimentos dependen de forma lineal de los contenidos en los mismos, sistemas lineales, o (2) que los flujos entre los compartimentos y sus contenidos no se relacionan de una manera lineal, sistemas no-lineales (Rhode 1992). Nuestros resultados muestran que al producirse una perturbación por deposición antropogénica de un metal traza como el cobre, tóxico en altas concentraciones y con alta afinidad por compuestos orgánicos, el sistema se comporta de manera no lineal. Lo anterior puede deberse a la complejidad de los procesos que controlan las transferencias entre componentes en los ecosistemas (Jorgensen 1994). En el caso del cobre, su transferencia desde una fuente externa, atmosférica, dependería de al menos dos variables controladoras actuando entre distintos componentes del ecosistema: la transferencia entre el MPD y el suelo superficial sería función de la proporción de materia orgánica en el MPD, en tanto que la transferencia entre el suelo y los componentes bióticos sería dependiente del pH del suelo. Procesos tales como la descomposición bacteriana que disminuyen la proporción de materia orgánica facilitarán la transferencia de cobre entre los horizontes, en tanto que una disminución del $\mathrm{pH}$, por ejemplo debido a la acidificación como resultado de las emanaciones de $\mathrm{SO}_{2}$ por parte de refinerías y fundiciones, aumentarán la disponibilidad de este para la vegetación. Debido a ello, un aumento de cobre en un componente (e.g. MPD), crecimiento ecosistémico sensu Ulanowicz (1987), no se traduce en aumentos proporcionales del elemento en todo el circuito intrasistémico. De hecho, si nuestra interpretación es correcta, un aumento en la concentración de cobre en el MPD generaría una potencial inhibición del crecimiento ecosistémico al disminuir los procesos de descomposición y mineralización. 
Ello refuerza la idea de que los procesos de perturbación antropogénica, al menos aquellos que potencialmente perturban ciclos de elementos, deberían ser analizados desde una perspectiva ecosistémica. Lo anterior debería incluir un estudio detallado de la distribución del elemento bajo estudio entre los diferentes componentes del ecosistema. En este sentido, el modelo del ciclo intrasistémico del cobre usado como base de este estudio (Fig. 1), es una herramienta útil para analizar la dinámica de este elemento en ecosistemas forestales.

\section{AGRADECIMIENTOS}

Este trabajo formó parte de la tesis para optar al grado de Magister en Ciencias Biológicas con mención en Ecología otorgada en la Facultad de Ciencias, Universidad de Chile de L. Delgado. El trabajo fue financiado por el proyecto Copper Contents in the Components of Mediterranean Ecosystem y por una beca del Servicio de Intercambio Académico de Alemania (DAAD) otorgada a L. Delgado. Los autores agradecen la asesoría de la Profesora María Inés Toral del Laboratorio de Química Analítica de la Facultad de Ciencias, Universidad de Chile y del Profesor Dr. Victor Marín del Laboratorio de Modelación Ecológica de la Facultad de Ciencias, Universidad de Chile.

\section{LITERATURA CITADA}

ALLOWAY BJ \& DC AYRES (1993) Chemical principles of environmental pollution. Blackie Academic \& Professional, New York, New York. 291 pp.

BARKMANN J, B BRECKLING, T POTTHAST \& J BADURA (1998) Introduction: Philosophical aspects of goal functions. En: Müller F \& M Leupelt (eds) Eco-targets, goal functions, and orientors: 289-297. Springer-Verlag, Berlin, Germany.

BESIER PM, J HOWELL \& G BRUNTLET (1994) Advanced electrochemical techniques versus atomic absorption spectrometry: inductively coupled plasma atomic emission mass spectrometry in environmental analysis. Analyst 119: 219-231.

BLEW RD (1996) On the definition of ecosystem. Ecological Society of America Bulletin 77: 171-173.

COX BR (1988) Sensitivity of forest plant reproduction to long-range transported air pollutants: the effects of wet deposited acidity and copper on reproduction of Populus tremuloides. New Phytology 10: 33-38.

DAEHLER CC \& D STRONG (1996) Can you bottle nature? The role of microcosms in ecological research. Ecology 77: 663-664.
DONOSO C (1993) Bosques templados de Chile y Argentina: variación, estructura y dinámica. Editorial Universitaria, Santiago, Chile. 484 pp.

DUVIGNEAUD P \& S DENAEYER-DE SMET (1970) Biological cycling of minerals in temperate deciduous forests. En: Jacobs J, O Lange, J Olson \& W Vieser (eds) Ecological studies I; temperate forest ecosystems: 199-225. Springer-Verlag, Berlin, Germany.

FAUTH JE (1997) Working toward operational definitions in ecology: putting the system back into ecosystem. Ecological Society of America Bulletin 78: 295-297.

FILANI GA (1976) Effects of different fungicidal copper compounds on Phytophthora palmivora. Turrialba 26: $295-301$

GINOCCHIO R (1996) Cuantificación de la tolerancia al cobre y al sulfato en dos especies leñosas de Chile central. Revista Chilena de Historia Natural 69: 413424.

GONZÁLEZ S (1992) Prospección de elementos trazas en recursos agrícolas de Chile. III. Suelos de los valles Mapocho y Maipo, Región Metropolitana. Agricultura Técnica (Chile) 52: 320-329.

HALLÉ F, R OLDEMANN \& P TOMLIMSON (1978) Tropical trees and forests: an architectural analysis. Springer-Verlag, Berlin, Germany. $441 \mathrm{pp}$.

HARPER J (1997) Population biology of plants. Academic Press, New York, New York. 892 pp.

KIIKKILÄ O, J PERKIÖMAKÏ, M BARNETTE, J DEROME, T PENNANCAN, E TULISALO \& $\mathrm{H}$ FRITZE (2001) In situ bioremedation through mulching of soil polluted by a copper-nickel smelter. Journal of Environmental Quality 30: 1134-1143.

KELLER C \& JC VÉDY (1994) Distribution of copper and cadmium fractions in two forest soils. Environmental Quality 23: 987-999.

KRAMER PA, D ZABOWSKI, G SCHERER \& RL EVERETT (2001) Native plant restoration of copper mine tailings. I. Substrate effect on growth and nutritional status in a Greenhouse study. Journal of Environmental Quality 29: 1762-1769.

JORGENSEN SE \& F MÜLLER (2000) Handbook of ecosystem theories and management. Lewis Publishers, London, United Kingdom. 584 pp.

JØRGENSEN S (1992) Integration of ecosystem theories. Kluwer Academic Publishers, Dordrecht, The Netherlands. 383 pp.

LASKOWSKI R, M KIKLINSKA \& M MARYANSKI (1995) The dynamics of chemical elements in copper and cadmium fractions in two forest soil: heavy metals in the environment. Ecology 76: 1393-1406.

LOBERLI EM \& E STEINNES (1988) Metal uptake in plants from a birch forest area near smelter in Norway. Water, Air \& Soil Pollution 37: 25-39.

LIKENS G (1992) An ecosystem approach: its use and abuse. Ecology Institute, Oldendorf/Luhe, Germany. $166 \mathrm{pp}$.

LOTKA A (1924) Elements of physical biology. Dover Publications, New York, New York. 465 pp.

MCBRIDE MB (1994) Environmental chemistry of soils. Oxford University Press, Oxford, United Kingdom. $406 \mathrm{pp}$. 
MARÍN VH \& GR OLIVARES (1999) Estacionalidad de la productividad primaria en Bahía Mejillones del Sur (Chile): una aproximación proceso-funcional. Revista Chilena de Historia Natural 72: 629-641.

MARÍN VH (1997) General system theory and the ecosystem concept. Bulletin of the Ecological Society of America 78: 102-104.

MOHANTY N, I VASS \& S DEMETER (1989) Copper toxicity photosystem II electron transport at the secondary quinone acceptor, $\mathrm{QB}^{1}$. Plant Physiology 90: 175-179.

MOONEY HA, E MEDINA, D SCHINDLER, E-D SCHULZE \& BH WALKER (1991) Ecosystem experiments. John Wiley \& Sons, New York, New York. 268 pp.

O'NEILL RV, DL ANGELIS, JB WAILDE \& TFH ALLEN (1986) A hierarchical concept of ecosystems. Princenton University Press, Princenton, New Jersey. $253 \mathrm{pp}$.

PÉREZ C (1994) Índices de esclerófilia en relación a la calidad química de la hojarasca y el grado de mineralización potencial del nitrógeno del suelo superficial del bosque de "olivillo" (Aextoxicon punctatum R. et Pav) en Chile. Revista Chilena de Historia Natural 67: 113-109.

RHODE H (1992) Modeling biogeochemical cycles. En: Butcher SS, RJ Charlson, GH Orians \& GV Wolfe (eds) Global biogeochemical cycles: 55-72. Academic Press, New York, New York.

ROWE JS (1997) Defining the ecosystem. Ecological Society of America Bulletin 78: 95-97.

SEREY I, R BUSTAMENTE, M TORAL, P RICHTER \& L YATES (1997) Copper contents in Mediterranean ecosystems of South America. Environmental Monitoring and Assessment 45:223-235.
SHIOI Y, H TAMAI \& T SASA (1978) Effects of copper on photosynthetic electron transport systems in spinach chloroplasts. Plant and Cell Physiology 19: 203-209.

SOKAL RR \& FJ ROHLF (1969) Biometry: the principles and practice of statistics in biological research. Second edition. W.H. Freeman \& Company, San Francisco, California. 859 pp.

STEVENSON FJ (1994) Humus chemistry: genesis, composition, reactions. John Wiley \& Sons, New York, New York. 496 pp.

TANSLEY A (1935) The use and abuse of vegetational concepts and terms. Ecology 16: 284-307.

TYLER G (1984) The impact of heavy metal a case study of pollution on forests: Gusum, Sweden. Ambio 12: $18-24$.

ULANOWICZ RE (1987) Growth and development: ecosystem phenomenology. Springer-Verlag, Berlin, Germany. 203 pp.

ULANOWICZ RE (1991) Formal agency in ecosystem development. En: Higashi M \& TP Burns (eds) Theoretical studies of ecosystems; the network perspective: 58-70. Cambridge University Press, Cambridge, United Kingdom.

WARING EH \& WH SCHLESINGER (1985) Forest ecosystems. Academic Press, Orlando, Florida. 340 pp.

ZUNINO H \& JP MARTIN (1977) Metal-Binding organic macromolecules in soil: 1. Hypothesis interpreting the role of soil organic matter in the translocation of metal ions from rocks to biological systems. Soil Science 123: 65-76.

ZHOU LX \& JWC WONG (2001) Effect and dissolved organic matter from sludge and sludge compost on soil copper sorption. Journal of Environmental Quality 30: $878-883$. 